\title{
A hybrid method combining the Time-Domain Method of Moments, the Time-Domain Uniform Theory of Diffraction and the FDTD
}

\author{
A. Becker and V. Hansen
}

Chair of Electromagnetic Theory, University of Wuppertal, Germany

\begin{abstract}
In this paper a hybrid method combining the Time-Domain Method of Moments (TD-MoM), the TimeDomain Uniform Theory of Diffraction (TD-UTD) and the Finite-Difference Time-Domain Method (FDTD) is presented. When applying this new hybrid method, thin-wire antennas are modeled with the TD-MoM, inhomogeneous bodies are modelled with the FDTD and large perfectly conducting plates are modelled with the TD-UTD. All inhomogeneous bodies are enclosed in a so-called FDTD-volume and the thin-wire antennas can be embedded into this volume or can lie outside. The latter avoids the simulation of white space between antennas and inhomogeneous bodies. If the antennas are positioned into the FDTD-volume, their discretization does not need to agree with the grid of the FDTD. By using the TD-UTD large perfectly conducting plates can be considered efficiently in the solution-procedure. Thus this hybrid method allows time-domain simulations of problems including very different classes of objects, applying the respective most appropriate numerical techniques to every object.
\end{abstract}

\section{Introduction}

Every numerical method has inherent advantages and disadvantages. For example the FDTD is not efficient for modelling electrically large but simple bodies whereas the TDUTD is well suited for such bodies but cannot be applied to inhomogeneous bodies. Consequently, by combining several techniques hybrid methods can be created to overcome the disadvantages and simultaneously make use of the advantages of the underlying techniques.

In the context of this paper we treat transient problems as shown in Fig. 1: Electrically large perfectly conducting plates are modelled with the TD-UTD, thin-wire antennas are modelled with the TD-MoM and inhomogeneous bodies are modelled with the FDTD. The aim of this paper is the hybridization of all three methods. Therefore, first of all

Correspondence to: A. Becker

(abecker@ieee.org)
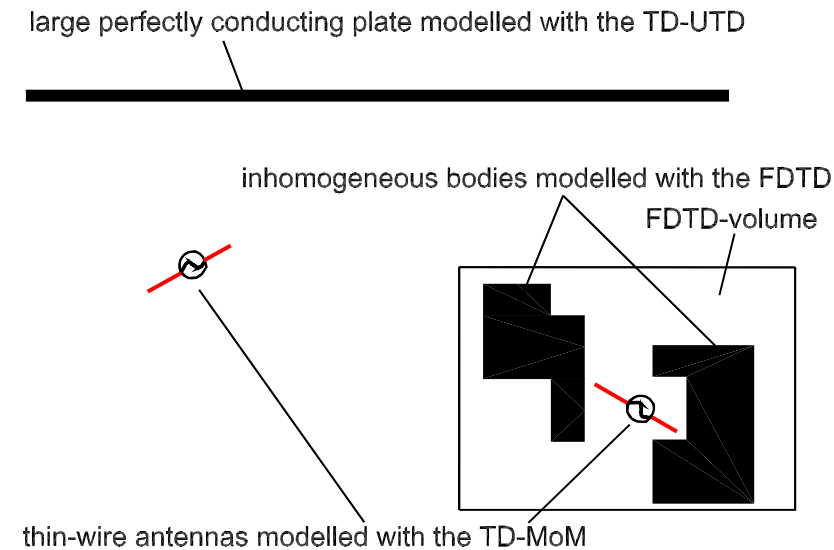

Fig. 1. Schematic representation of the proposed hybrid method.

the three methods are shortly presented in Sect. 2-4 without claiming to give a complete overview about state of the art developments of the specific methods, but pointing out the properties, which are important for the hybridization. In Sect. 5 the hybrid method combining all three methods is developed step by step. Finally, numerical examples are presented.

\section{TD-MoM}

Considered are perfectly conducting thin-wire antennas (surface $S$ ) located in free space. One standard integral equation for the electric field radiated in free space by the current density $\boldsymbol{J}$ flowing on the surface $S$ reads:

$\boldsymbol{E}^{\mathrm{rad}}(\boldsymbol{r}, t)=\int_{S}\left[-\nabla \frac{\rho\left(\boldsymbol{r}^{\prime}, t\right)}{4 \pi \varepsilon r}-\frac{\partial}{\partial t} \frac{\mu \boldsymbol{J}\left(\boldsymbol{r}^{\prime}, t^{\prime}\right)}{4 \pi r}\right] d a^{\prime}$,

with $r=\left|\boldsymbol{r}-\boldsymbol{r}^{\prime}\right|, \quad \rho\left(\boldsymbol{r}^{\prime}, t\right)=-\int_{0}^{t^{\prime}} \nabla \boldsymbol{J}\left(\boldsymbol{r}^{\prime}, t^{\prime}\right) d t$ and $t^{\prime}=t-\frac{r}{c}$. For the numerical solution the Ansatz

$\boldsymbol{J}\left(\boldsymbol{r}^{\prime}, t\right)=\sum_{i=1}^{N_{s}} \sum_{j=1}^{N_{t}} I_{i, j} \boldsymbol{\beta}_{i}\left(\boldsymbol{r}^{\prime}\right) \mathcal{T}_{j}(t)$ 
large perfectly conducting plate modelled with the TD-UTD

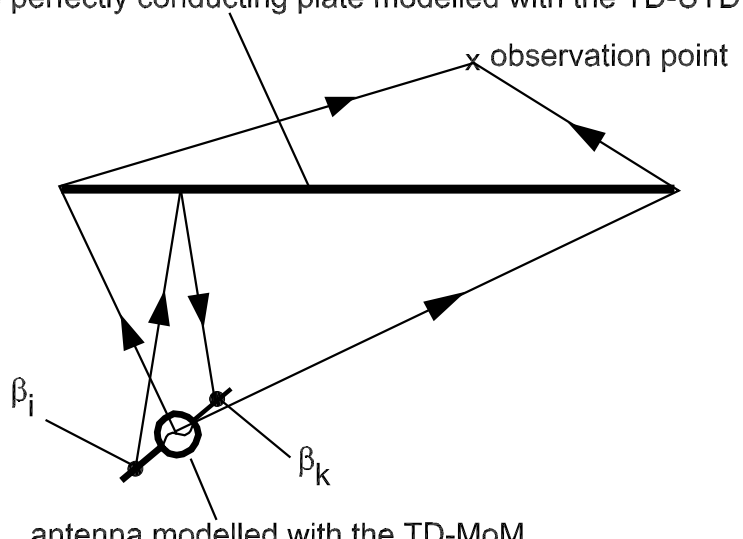

antenna modelled with the TD-MoM

Fig. 2. Schematic representation of the TD-MoM+TD-UTDHybrid Method. The diffracted field in an observation point is modelled using one equivalent source point. Additionally shown: One ray reflected from basis function $\boldsymbol{\beta}_{i}$ to test function $\boldsymbol{\beta}_{k}$.

is chosen to approximate the spatial and temporal dependency of the electric current density (Gómez et al., 1992). $N_{t}$ and $N_{s}$ denote the number of temporal and spatial basis functions, respectively and $I_{i, j}$ are unknown weighting coefficients. Substituting Eq. (2) into Eq. (1) and taking the temporal derivate of the electric field yields

$$
\begin{aligned}
\frac{\partial}{\partial t} \boldsymbol{E}^{\mathrm{rad}}(\boldsymbol{r}, t)= & \sum_{i=1}^{N_{s}} \sum_{j=1}^{N_{t}} \frac{I_{i, j}}{4 \pi} \int_{S_{i}}\left[\frac{\nabla \gamma_{i}\left(\boldsymbol{r}^{\prime}\right) \mathcal{T}_{j}\left(t^{\prime}\right)}{\varepsilon r}\right. \\
& \left.-\frac{\partial^{2}}{\partial t^{2}} \frac{\mu \boldsymbol{\beta}_{i}\left(\boldsymbol{r}^{\prime}\right) \mathcal{T}_{j}\left(t^{\prime}\right)}{r}\right] d a^{\prime},
\end{aligned}
$$

where $\gamma_{i}\left(\boldsymbol{r}^{\prime}\right)=\nabla \boldsymbol{\beta}\left(\boldsymbol{r}^{\prime}\right)$. A scalar equation is derived by multiplying Eq. (3) with a test function $\boldsymbol{\beta}_{k}$ and integrating the result over the domain $S_{k}$ of the test function. In a next step, the time axis is subdivided into time steps $t=n T$ and it is assumed that

$\mathcal{T}_{j}(t)=\left\{\begin{array}{cl}0 & \text { if }|t-j T| \geq T \\ \mathcal{T}(t-j T) & \text { if }|t-j T|<T\end{array}\right.$

and that for $t_{0} 7=n_{0} T$ all coefficients $I_{i, j<n_{0}}$ are known. On the surface of perfectly conducting scatterers the total tangential electric field must vanish, i.e. the sum of the incoming fields $\boldsymbol{E}^{\text {inc }}$ and the radiated fields $\boldsymbol{E}^{\text {rad }}$ must be zero. Fulfilling this boundary condition at the domain of all test functions $\boldsymbol{\beta}_{k}$ and for $t_{0}$ leads to a systems of linear equations (Gómez et al., 1992) to calculate the coefficients $I_{i, n_{0}}$. Consequently, in a so-called marching-on-in-time procedure all coefficients $I_{i, j}$ can be calculated successively. The potential consider incoming field in the solution procedure is the main point of contact for hybridization.

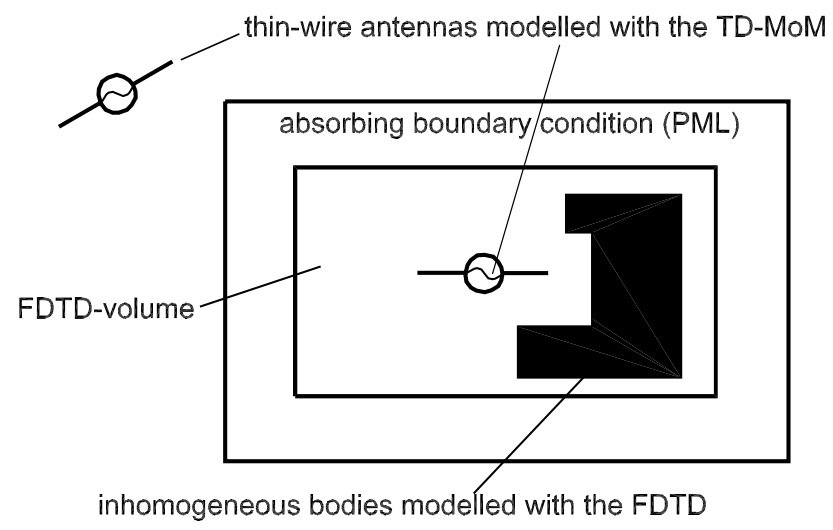

Fig. 3. Schematic representation of the TD-MoM+FDTD-Hybrid Method.

\section{Time-domain Geometrical Theory of diffraction}

The Geometrical Theory of Diffraction is based on the representation of waves by rays. According to the UTD, the field radiated by a point-source in presence of a perfectly conducting plate can be approximated as the sum of the direct ray, a reflected ray and diffracted rays (Rousseau and Pathak, 1995):

$\boldsymbol{E}=\boldsymbol{E}_{L O S} U_{i}+\boldsymbol{E}_{R} U_{R}+\boldsymbol{E}_{D}$,

with $U_{i}=1$ if line of sight (LOS) is given between source and observation point and $U_{i}=0$ if no direct ray reaches the observation point. $U_{R}$ is equal to one, if a reflected ray reaches the observation point and it is equal to zero, otherwise. According to the UTD, the reflected and diffracted fields are found by using the fields in the reflection and diffraction points, respectively. Both $U_{i}$ and $U_{R}$, as well as possible reflection and diffraction points are found by using a raytracing algorithm.

The diffracted field is found by convolution of the field $\mathbf{E}_{D_{0}}$ in the diffraction point with the diffraction coefficient and multiplying the result with the spreading factor $A\left(s_{d}\right)$ :

$\mathbf{E}_{D}\left(s_{d}, t\right)=\mathbf{E}_{D_{0}}\left(t-\frac{s_{d}}{c}\right) * \stackrel{\leftrightarrow}{d}\left(t-\frac{s+s_{d}}{c}\right) A\left(s_{d}\right)$,

with

$A\left(s_{d}\right)=\sqrt{\frac{s^{\prime}}{s_{d}\left(s_{d}+s^{\prime}\right)}}$.

The dyadic diffraction coefficient $\stackrel{\leftrightarrow}{d}(t)$ can be found in Rousseau and Pathak (1995). In the context of this paper, the fields in the reflection and diffraction points are calculated with the TD-MoM and the FDTD, respectively. Thus these fields act as point of contact for hybridization. 


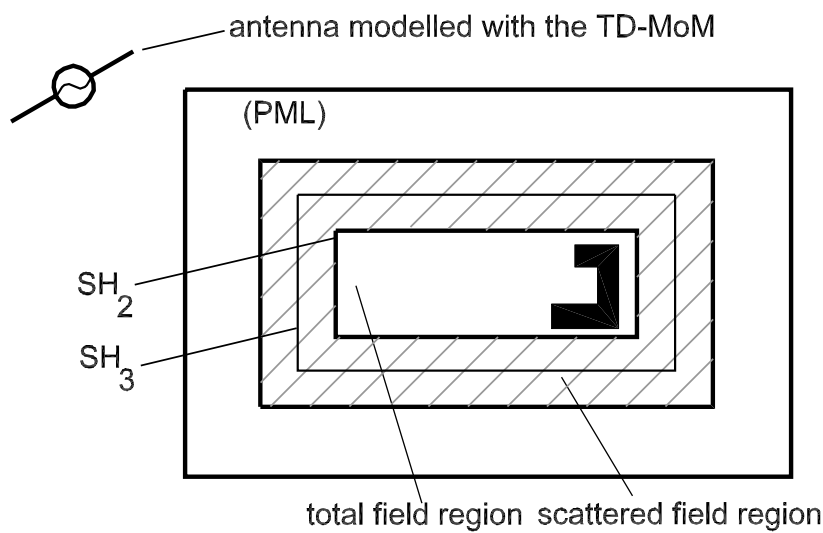

Fig. 4. Subdivision of the FDTD-volume into total field region and scattered field region and the Huygens surfaces used for hybridization.

\section{FDTD}

For this paper a simple 3D-FDTD with a Perfectly Matched Layer (PML) as absorbing boundary condition is used; for details see e.g. Taflove (2000). The points of contact for hybridization are the total-field/scattered-field formulation (see e.g. Huang et al., 1999) to introduce incoming fields into the FDTD-volume and the calculation of fields outside the FDTD-Volume with a surface integral representation of the electric field (see e.g. Skarlatos et al., 2005).

\section{Hybrid methods}

The three methods are hybridized by using well known theorems and making use of the superposition principle. In the following - for the sake of simplicity - the process of hybridization is described step by step.

\subsection{TD-MoM+TD-UTD-Hybrid Method (Becker and Hansen, 2006)}

The schematic representation of this hybrid method is shown in Fig. 2. The hybridization of both methods comprises two steps:

1. The fields in the observation points are calculated with Eq. (5). For this purpose, the fields radiated by the thinwire antennas are approximated by rays with source points lying on the surface $S$ of the antennas, which are modelled with the TD-MoM. In many cases the rays reaching an observation point can be constructed by using one equivalent source-point in the centre of the antenna (see Fig. 2). The direct fields in the observation points (if line of sight is given, i.e. $U_{i} \neq 0$ ) and the fields in the reflection and diffraction points are calculated in the same marching-on-in-time procedure as the currents

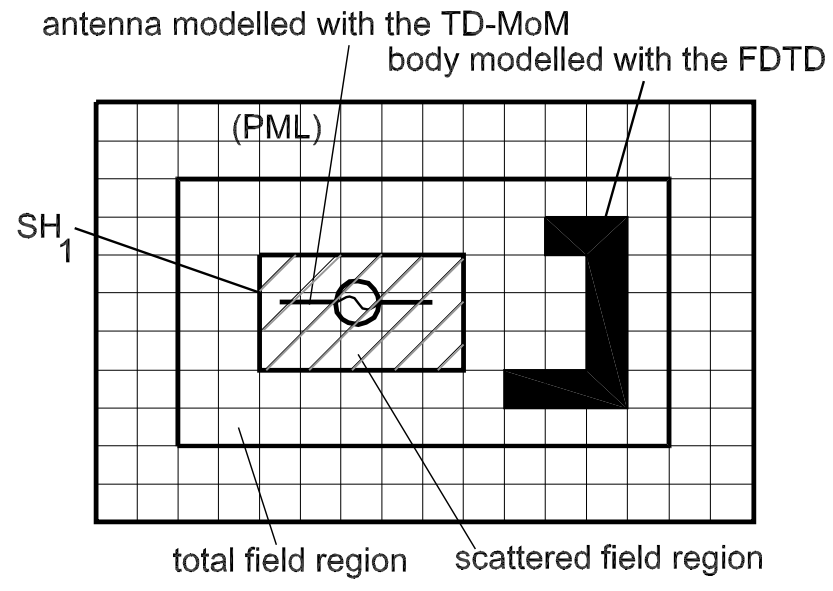

Fig. 5. Subdivision of the FDTD-volume into total field region and scattered field region and the Huygens surfaces used for hybridization.

flowing on the antenna. All necessary current coefficients $I_{i, j}$, which excite a field at $t=n_{0} T$ in the observation point are - due to the distance between source and observation point - calculated in the marching-onin-time procedure before.

2. The influence of the surrounding on the currents is considered by treating reflected or diffracted fields, which reach the surface $S$ of the antennas, as incoming fields $\boldsymbol{E}^{\mathrm{inc}}$ in the marching-on-in-time procedure. Thus the free space Green's function used for Eq. (1) is implicitly modified. This strategy is well proven as it has been successfully applied for several frequency-domain hybrid methods (Alaydrus et al., 2002). Reflected and scattered fields are constructed by using the centre of any base function $\boldsymbol{\beta}_{i}$ as origin of the respective rays. For one infinitely large reflecting plate, $N_{s}$ basis functions and $N_{s}$ test functions this results into $N_{s}^{2}$ reflected rays. Consequently, this becomes relatively costly.

\subsection{TD-MoM+FDTD-Hybrid Method}

As shown above, the consideration of the influence of the surrounding on the currents flowing on the thin-wires is relatively costly. Additionally, the TD-MoM+TD-UTD Hybrid Method is restricted to problems involving simply structured scatterers. In the context of this paper, this restriction is overcome by using a TD-MoM+FDTD Hybrid Method. When applying this hybrid method, thin-wire antennas are modelled with the TD-MoM and inhomogeneous bodies are modelled with the FDTD. All inhomogeneous bodies lie inside the so-called FDTD-volume, which is enclosed by a PML (see Fig. 3). The TD-MoM is fully hybridized with the FDTD, if the fields radiated by the thin-wire antennas are considered in the FDTD solution-procedure (at the location of the inhomogeneous bodies) and if the influence of the 


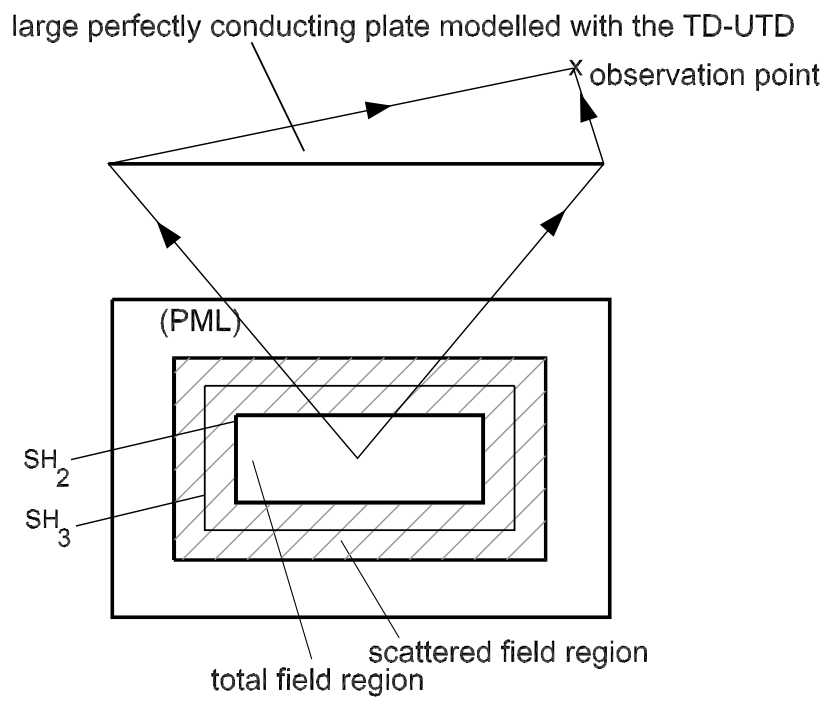

Fig. 6. Schematic representation of the TD-UTD+FDTD-Hybrid Method. Not shown: Rays backscattered to the FDTD-volume.

inhomogeneous bodies on the currents flowing on the thinwire antennas are considered in the TD-MoM solution procedure and vice versa. This is achieved by using the approach described in Huang et al. (1999): The basic idea is to subdivide the FDTD-region into a scattered field region and into a total field region. By this a numeric Huygens source scheme is realised and it is possible to introduce arbitrary incident fields into the FDTD-volume. For the sake of simplicity it is useful to use the following case differentiation:

\subsubsection{Thin-wire antennas outside the FDTD-volume}

Figure 4 shows the case of an antenna modelled with the TDMoM, which lies outside the FDTD-Volume. The fields radiated by the thin-wire antennas are introduced into the FDTDsolution procedure by using the surface $\mathrm{SH}_{2}$, which seperates the total field region (inside $\mathrm{SH}_{2}$ ) from the scattered field region (outside $\mathrm{SH}_{2}$ ). Consequently, at the position of the inhomogeneous bodies, the unknowns of the FDTD include the fields radiated by the thin-wire antennas. The fields radiated or scattered by the FDTD-volume are calculated by using equivalent sources on the Huygens surface $\mathrm{SH}_{3}$ lying just inside the PML (see Fig. 4) and a surface integral representation of the electric field (see e.g. Taflove, 2000). These fields are used as incoming fields $\boldsymbol{E}^{\text {inc }}$ in the TD-MoM solution procedure.

\subsubsection{Thin-wire antennas inside the FDTD-volume}

As shown in Fig. 5, the antennas modelled with the TD-MoM now lies inside the FDTD-Volume. The FDTD-volume is seperated by the Huygens surface $S H_{1}$ into two regions: A total field region and a scattered field region. The scattered field region needs to be homogeneous and includes the anten-

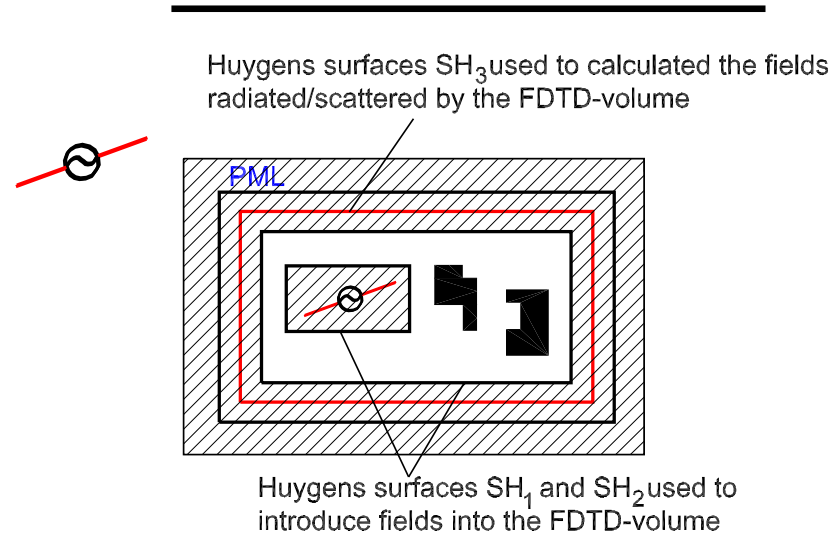

Fig. 7. The three necessary Huygens surfaces for full hybridization of all three methods.

nas modelled with the TD-MoM. The discretiziation of the latter does not need to agree with the grid of the FDTD. The total field region includes the inhomogenous bodies and can include arbitrary material (see Fig. 5). The scattered field, which is in fact the field back-scattered to the antenna by its surrounding, is used as incoming field in the TD-MoM solution procedure. As the antenna lies inside the FDTD-volume, fields outside the FDTD-volume can be calculated by using $\mathrm{SH}_{3}$ (not shown in Fig. 5) and the procedure described above. Consequently, for observation points outside the FDTD-volume, the FDTD+TD-MoM Hybrid-Method does not distinguish from a stand-alone FDTD method.

\subsection{TD-UTD+FDTD-Hybrid Method}

The FDTD is hybridized with the TD-UTD by using a strategy very similar to Skarlatos et al. (2005): In the application of the TD-UTD the fields in the reflection and diffraction points, as well as the direct fields, are calculated using once again a surface integral representation and the equivalence principle (surface $\mathrm{SH}_{3}$ ). To save memory and CPUtime $\mathrm{SH}_{3}$ is subdivided into large subdomains and for the ray-representation of the fields one equivalent source point per subdomain is used. Obviously, the farther the reflection and diffraction points are away from the FDTD-volume, the less subdomains are needed. Therefore it is sometimes useful, to use one equivalent source point in the centre of the FDTD-volume as shown in Fig. 6. Fields back-scattered to the FDTD-volume can be taken into account in the FDTD solution procedure by using the same procedure and the same surface $\mathrm{SH}_{2}$ as described in Sect. 5.2.1.

\subsection{TD-MoM+TD-UTD+FDTD-Hybrid Method}

When applying this hybrid method thin-wire antennas are modelled with the TD-MoM, large perfectly conducting plates are modelled with the TD-UTD and inhomogeneous bodies are modelled with the FDTD. The hybridization of 


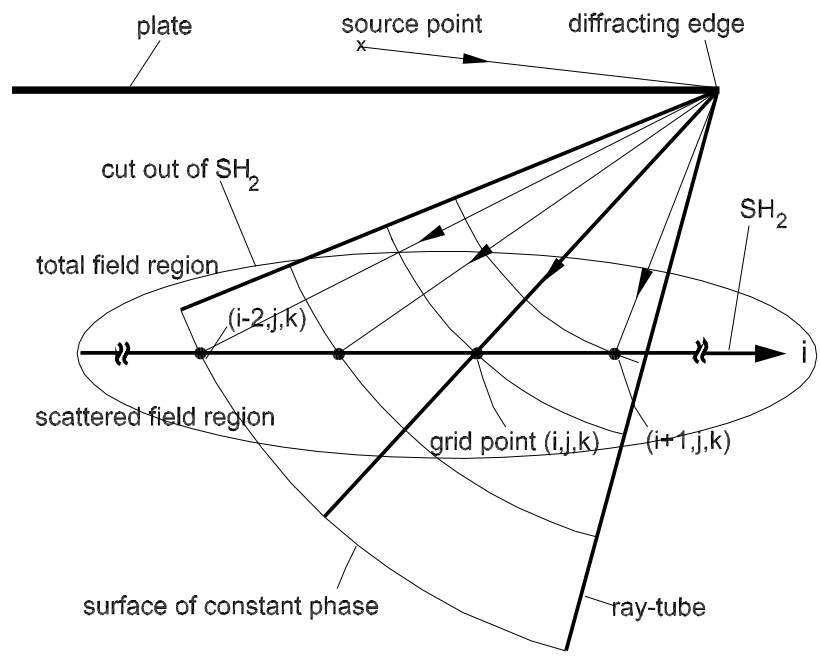

Fig. 8. Rays reaching a cut out of $\mathrm{SH}_{2}$ and a ray tube enclosing 4 grid points.

all three methods is achieved by starting with the TDMoM+TD-UTD hybrid method and using the superposition principle. The TD-MoM+TD-UTD hybrid method considers the interactions between the thin-wire antennas and the large plates. Additional FDTD bodies require the use of the Huygens surfaces $\mathrm{SH}_{1}$ and $\mathrm{SH}_{2}$ to introduce the fields radiated by the thin-wire antennas into the FDTD-volume. These fields can include fields, which are radiated by the thin-wire antennas and then scattered by the plates. $\mathrm{SH}_{3}$ is used to calculate the fields radiated or scattered by the FDTD-volume and these fields are considered as incoming fields $\boldsymbol{E}^{\text {inc }}$ in the TD-MoM solution procedure. Additionally, these fields $\boldsymbol{E}^{\text {inc }}$ can include fields radiated by the FDTD-volume and then scattered by the plates. Thus the interactions between the thin-wire antennas and the inhomogeneous bodies are considered including the additional influence of the plates. Consequently, one needs to use all three Huygens surfaces $S H_{1}$, $\mathrm{SH}_{2}$ and $\mathrm{SH}_{3}$ and by this all three methods are hybridized (see Fig. 7).

\subsection{Major drawbacks and improvement opportunities}

One of the main drawbacks of the proposed methods are the resource and calculation requirements to calculate the fields radiated by the FDTD-volume. These fields are calculated by using equivalent sources on the Huygens-surface $\mathrm{SH}_{3}$ and an integral representation of the electric field. This calculation is very costly and we assume that these costs can be reduced by using techniques like the one proposed in Oetting and Klinkenbusch (2005).

Right now, the second main drawback is the fact that the fields scattered by the plates have to be calculated pointwise (see also Sect. 2), in order to be introduced into the FDTDvolume and to be used as incoming field in the TD-MoM

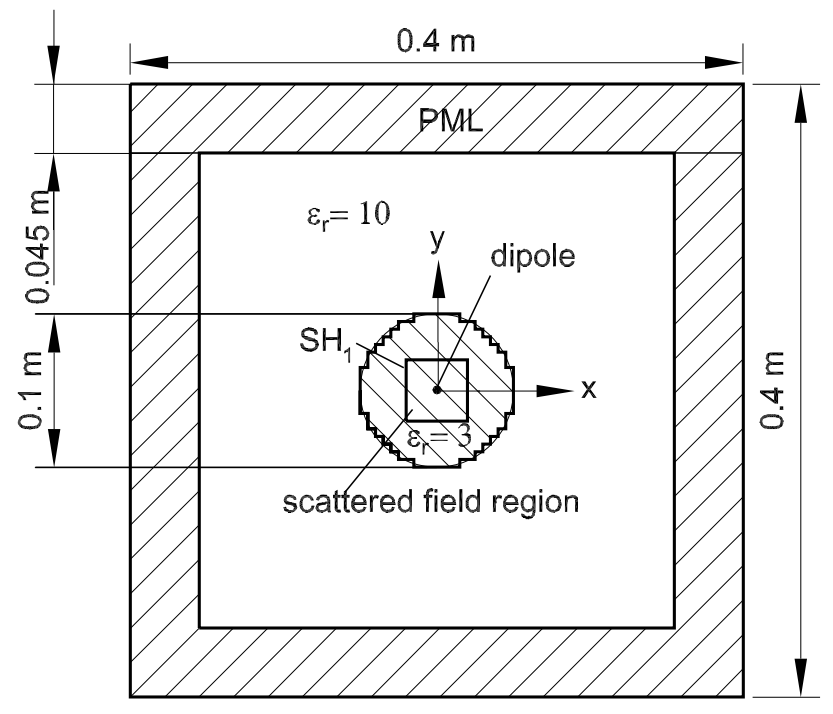

Fig. 9. Dipole embedded into a dielectric cylinder.

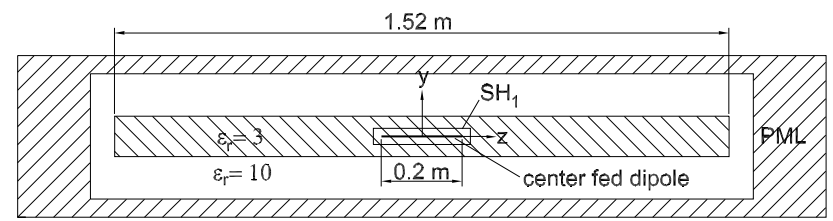

Fig. 10. Dipole embedded into a dielectric cylinder.

solution procedure. That means, that for any grid point on the surface $\mathrm{SH}_{2}$ individual rays have to be constructed (see Fig. 8). This is extremely costly but in future developments this might be accelerated by making more use of the ray representation of the scattered fields. As indicated in Fig. 8 ray tubes can be constructed, which enclose more than one grid point and by this the cost for constructing the rays becomes much smaller. The drawback of this strategy is that the temporal behaviour of the field in one reference point (grid point $(i, j, k)$ in Fig. 8) has to be stored in memory to be able to calculate the fields on different surfaces of constant phase.

\section{Numerical examples}

\subsection{Dipole embedded into a dielectric cylinder}

Considered is the geometry shown in Fig. 9 and Fig. 10, consisting of a center-fed dipole (radius $r=0.001 \mathrm{~m}$ ) embedded into a dielectric cylinder, which is surrounded by a dielectric medium. In Fig. 11 the input impedance of the dipole is shown. The result calculated with the TD-MoM+FDTDHybrid Method is compared to the result calculated with a frequency-domain method of moments for cylindrically layered media (Becker and Hansen, 2006). 


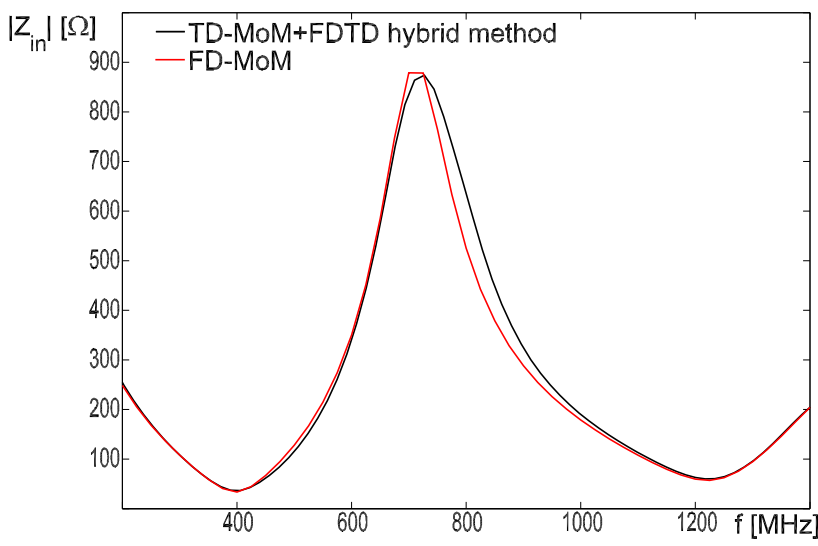

Fig. 11. Input impedance calculated with the TD-MoM+FDTD Hybrid Method and with the frequency domain Method of Moments.

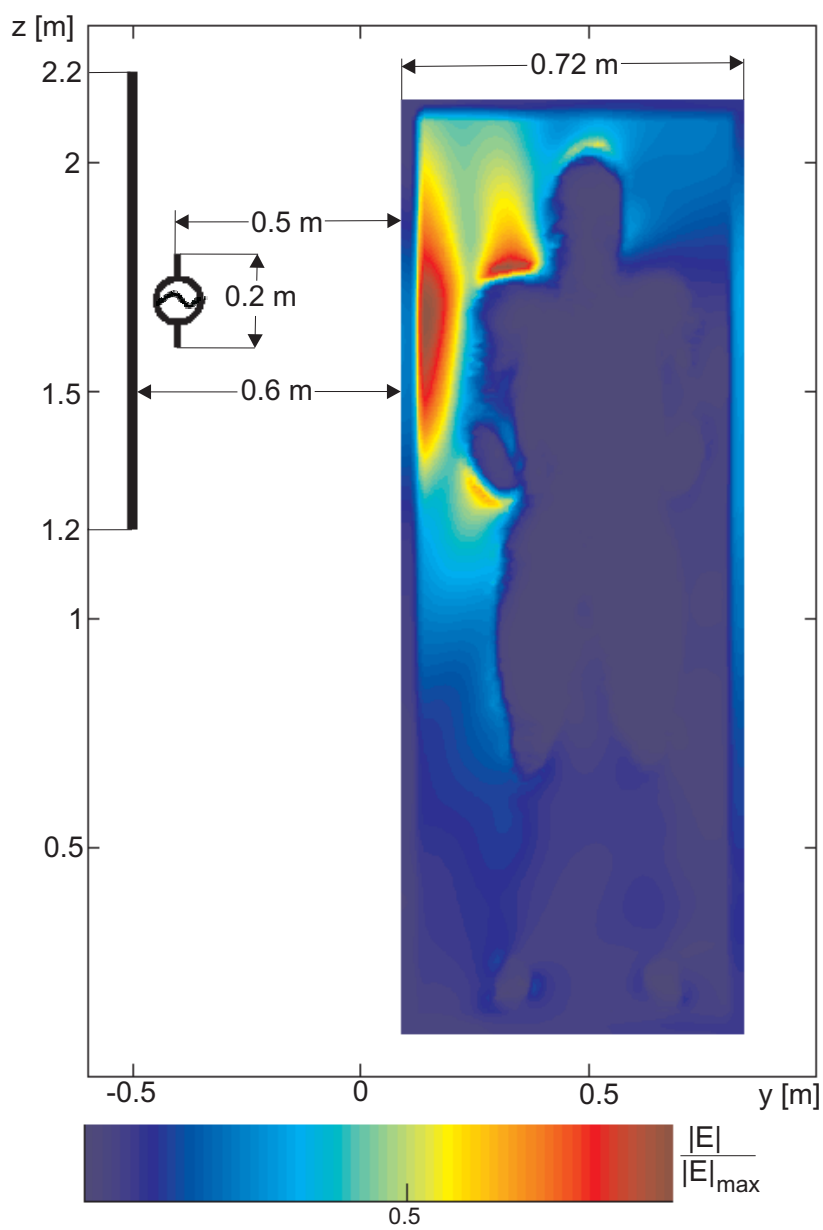

Fig. 12. Human body in front of a center-fed dipole and a perfectly conducting plate.

\subsection{Human body in front of a dipole with reflector}

As a second example, a center-fed dipole $(r=0.001 \mathrm{~m})$ in front of a perfectly conducting plate (infinitely long in $\pm x$ direction) and a model of the human body is considered (based on visible human National Library, 1986; Gabriel, 2006, discretized using $\triangle_{x}=\triangle_{y}=\triangle_{z}=0.01 \mathrm{~m}$ ). In Fig. 12 the absolute value of the electric field inside the body (cut at $x=0.31 \mathrm{~m}$, i.e. the plane including the dipole) is shown. For this calculation diffraction at the edges and corners of the plate and the influence of the human body towards the currents flowing on the dipole are neglected. The fields radiated by the thin-wire antennas and reflected by the plate are calculated by using mirror sources.

\section{Conclusions}

The presented method combines three techniques to a new hybrid method. Using this it is possible to aply appropriate numerical techniques for modelling different types of bodies: The TD-UTD for large, perfectly conducting bodies, the TDMoM for small, perfectly conducting bodies and the FDTD for dielectric bodies. The combination of these three approaches yields a powerful tool for handling problems which cannot be treated with any other time-domain technique.

\section{References}

Alaydrus, M., Hansen, V., and Eibert, T. F.: Hybrid ${ }^{2}$ : Combining the three-dimensional hybrid finite element-boundary integral technique for planar multilayered media with the uniform geometrical theory of diffraction, IEEE Trans. Antennas Propagat, vol. 50, pp. 67-74, Jan., 2002.

Becker, A. and Hansen, V.: A Hybrid Method Combining the MultiTemporal Resolution Time-Domain Method of Moments with the Time-Domain Geometrical Theory of Diffraction for ThinWire Antenna Problems, IEEE Trans. Antennas Propagat, vol. 54, pp. 953-960, March, 2006.

Becker, A., Zhou, Y., and Hansen, V.: On the Calculation of the Radiation Properties of Dipole Antennas in Cylindrical Boreholes Considering Large Scatterers, German Microwave Conference, Karlsruhe, 2006.

Gabriel, C.: Brooks Air Force Technical Report AL/OE-TR-19960037, http://www.fcc.gov/fcc-bin/dielec.sh.

Gómez, R., Martin, A., and Rubio Bretones, A.: Time-domain integral equation methods for transient analysis, IEEE Magazine Antennas Propagat, vol. 34, pp. 15-23, June, 1992.

Huang, Z., Demarest, K. R., and Plumb, R. G.: An FDTD/MoM hybrid technique for modeling complex antennas in the presence of heterogeneous grounds; Geoscience and Remote Sensing, IEEE Trans. Antennas Propagat, vol. 37, pp. 2692-2698, Nov., 1999.

National Library of Medicine: Visible Human Project: http://www. nlm.nih.gov/research/visible/visible_human.html, 1986.

Oetting, C.-C. and Klinkenbusch, L.: Near-to-Far-Field Transformation by a Time-Domain Spherical-Multipole Analysis, Trans. AP, vol. 53, pp. 2054-2063, 2005. 
Rousseau, P. R. and Pathak, P. H.: Time domain geometrical theory of diffraction for a curved wedge, IEEE Trans. Antennas Propagat, vol. 43, pp. 1375-1382, Dec., 1995.

Skarlatos, A., Schuhmann, R., and Weiland, T.: Solution of Radiation and Scattering Problems in Complex Environments Using a Hybrid Finite Integration Technique - Uniform Theory of Diffraction Approach, IEEE Trans. Antennas Propagat, vol. 53, pp. 3347-3357, October, 2005.
Taflove, A.: Computational Electrodynamics, Artech House, Boston, 2000. 